\title{
Draft genome sequences of Salmonella Oslo isolated from seafood and its laboratory generated auxotrophic
}

\section{mutant}

\author{
Kadeeja Jazeela1 ${ }^{1}$, Anirban Chakraborty ${ }^{1}$, Praveen Rai ${ }^{1}$, Ballamoole Krishna Kumar ${ }^{1}$, Shabarinath \\ Srikumar ${ }^{2}$, Scot van Nguyen ${ }^{2}$, Daniel Hurley², Seamus Fanning ${ }^{2}$, Indrani Karunasagar ${ }^{1}$ and Vijaya Kumar \\ Deekshit ${ }^{\circledR}$
}

1. Nitte University Center for Science Education and Research, Nitte (Deemed to be University), Deralakatte, Mangaluru - 575018, Karnataka, India.

2. University College Dublin, Food Safety and zoonoses, Dublin, Ireland.

$\triangle$ Corresponding author: Vijaya Kumar Deekshit, Ph.D. Assistant Professor; Nitte University Center for Science Education and Research (NUCSER), Kotekar Beeri Road, Paneer campus, Deralakatte, Nitte (Deemed to be University), Mangalore-575018, India, Phone no: +91 9880849437, Fax no: +91-8242204300/301/302, E-mail: deekshit1486@nitte.edu.in.

(C) The author(s). This is an open access article distributed under the terms of the Creative Commons Attribution License (https://creativecommons.org/licenses/by/4.0/). See http://ivyspring.com/terms for full terms and conditions.

Received: 2019.09.07; Accepted: 2019.11.07; Published: 2020.01.01

\begin{abstract}
In recent years, the concept of bacteria-mediated cancer therapy has gained significant attention as an alternative to conventional therapy. The focus has been on non-typhoidal Salmonella (NTS), particularly S. Typhimurium, for its anti-cancer properties, however, other NTS serovars such as Salmonella Oslo, which are associated with foodborne illnesses could potentially be effective anti-cancer agents. Here, we report the draft genome sequence of Salmonella Oslo isolated from seafood and its laboratory generated auxotrophic mutant.
\end{abstract}

Key words: Non typhoidal Salmonella, Salmonella Oslo, Bacteria mediated cancer therapy, whole genome sequence

\section{Introduction}

Non-typhoidal Salmonella (NTS) is one of the pathogens that frequently cause foodborne infections throughout the world. The pathogenic potential of NTS strains have been well understood [1]. However, its therapeutic potential as an anti-cancer agent remain unexplored. Among more than 2500 NTS serovars, S. Typhimurium VNP20009 [2-4], an auxotrophic mutant strain of $S$. Typhimurium A1-R $[5,6]$, has been successfully studied and was even tested in phase I clinical trial for the treatment of solid tumors [7]. Many features of Salmonella namely the ability to thrive in hypoxic environment of the tumor, to induce innate immune response against tumor or its bactofection, and ability to release anticancer genes within tumor were found to be favorable for tumor regression in animal models [8-10]. However, despite the success observed in animal models, the clinical trials didn't yield expected results. This prompted the researchers to look for alternative NTS strains for their anticancer properties. In this study, the aim was to sequence the genomes of Salmonella Oslo isolated from seafood and its laboratory generated auxotrophic mutant. The availability of genetic information provides a basis for further studies, particularly for investigating their role as anti-cancer agents.

Salmonella Oslo was isolated from seafood (squid sample) as per the protocol recommended by the FDA Bacteriological Analytical Manual [11] with minor modifications. Briefly, the seafood sample was pre-enriched in lactose broth, followed by enrichment in selenite cysteine broth and tetrathionate broth. Post enrichment, the sample was streaked on Hektoen enteric agar (HiMedia Laboratory Pvt Ltd, India). During the enrichment and plating steps, the incubation temperature was maintained at $37^{\circ} \mathrm{C}$ for 
16 to $18 \mathrm{~h}$. Colonies with specific morphological features were selected and were subjected to a series of biochemical tests such as indole test, methyl red test, Voges-Proskuer test, citrate test, triple sugar iron agar (TSIA) test, urease and lysine iron agar (LIA) test for conventional identification. The biochemically positive colonies were further confirmed by PCR using genus-specific primer inv $A$ [12]. Serotyping was done at National Salmonella and Escherichia Centre, Central Research Institute, Kasauli, India.

Lambda red recombinase method [13] was used to generate the auxotrophic mutant of Salmonella Oslo by inducing deletions in $\operatorname{argH}$ and leuB genes, which code for arginine and leucine respectively. Biofilm assay was performed using the method described by Stepanovic et al (2004) [14].

To compare the growth kinetics of Salmonella Oslo (SO1-wild type) and its mutant (LAT9), a $100 \mu \mathrm{l}$ aliquot of overnight culture of SO1 and LAT9 was added to $5 \mathrm{ml}$ Luria Bertani broth (HiMedia Laboratory Pvt Ltd, India) and incubated at $37^{\circ} \mathrm{C}$ with shaking at $200 \mathrm{rpm}$. The optical density was measured at $600 \mathrm{~nm}\left(\mathrm{OD}_{600}\right)$ at different time points such as 0,1 , 2 to $24 \mathrm{~h}$ after incubation and expressed as $\log \left(\mathrm{OD}_{600}\right.$ X 1000).

To sequence the genome of SO1 and its laboratory generated mutant, bacterial genomic DNA was extracted using a QIAamp DNA mini kit (Qiagen, Germany). The quality of the extracted DNA was checked by Qubit ${ }^{\circledR}$ and further verified by a bioanalyzer (Agilent technologies). The genomic DNA library was prepared using a Nextera XT DNA library preparation kit (Illumina, Inc, Cambridge, UK). The whole genome sequencing was performed at UCD, Dublin. The raw sequence data were generated using the Illumina MiSeq platform with a depth of 100x. The obtained paired end reads were merged and the genome was assembled using CLC genomics (version 11) [15]. The processed reads were aligned to the reference genome LT2 strain (LT571437) with Bowtie 2 program. The annotation and gene prediction of draft genome was done using the Rapid Annotations Subsystems Technology (RAST) (http:// rast.nmpdr.org/) [16].

The identification of the isolate as true Salmonella enterica serovar Oslo was confirmed by serotyping experiments. As expected, the auxotrophic strain (LAT9) was found to be phenotypically mutant to amino acid arginine and leucine. However, when PCR was performed to confirm the changes in the target genes $(\operatorname{argH}$ and $l e u B)$, it revealed no deletions in $\operatorname{argH}$ and leuB genes. This strange observation prompted us to determine the whole genome sequence of wild (SO1) and mutant (LAT9) strain. The generated library produced a total of 1193762 and 1221694 reads for wild type and mutant respectively. The paired end reads of SO1 were assembled into 121 contigs with coverage of 100x. The genome size was calculated at $4,860,262$ bp comprising of 4,974 protein coding genes. The GC content of this strain was found to be $52.2 \%$. The analysis obtained from the RAST also revealed 401 subsystems (Fig. 1). The annotated genome had 383 amino acid biosynthesis genes including $\operatorname{argH}$ and leuB. In addition, 78 tRNAs, 11 ncRNAs, 168 pseudo genes were also identified.

Similarly, the paired end reads of LAT9 were assembled into 199 contigs with coverage of 100x. The genome size was calculated at $4,890,414$ bp comprising of 5,082 protein coding genes. The GC content of this strain was found to be $52.2 \%$. The analysis obtained from the RAST also revealed 402 subsystems (Fig. 2). The annotated genome had 392 amino acid biosynthesis genes. 79 tRNAs, 11 ncRNAs, 239 pseudo genes were also identified.
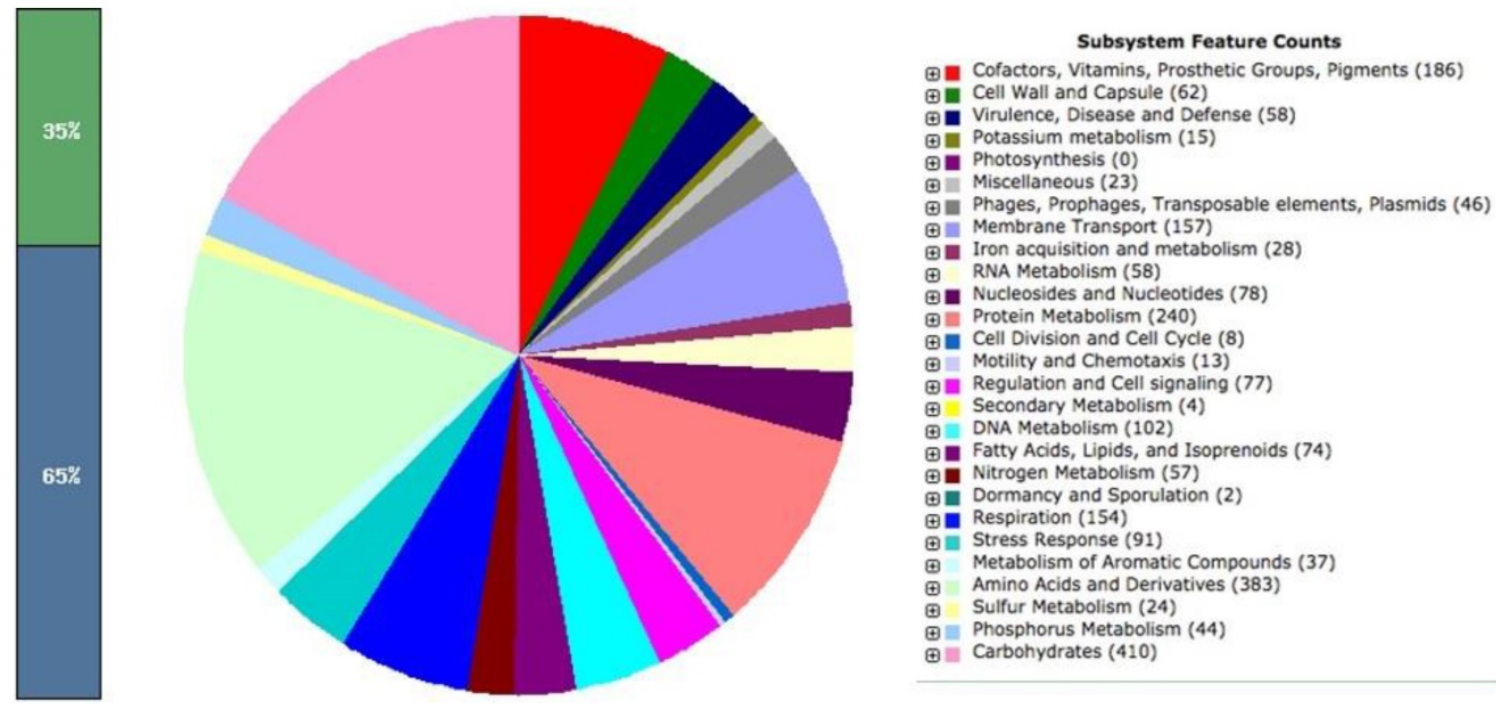

Figure 1. Salmonella Oslo (SOI)-WT subsystem feature. 

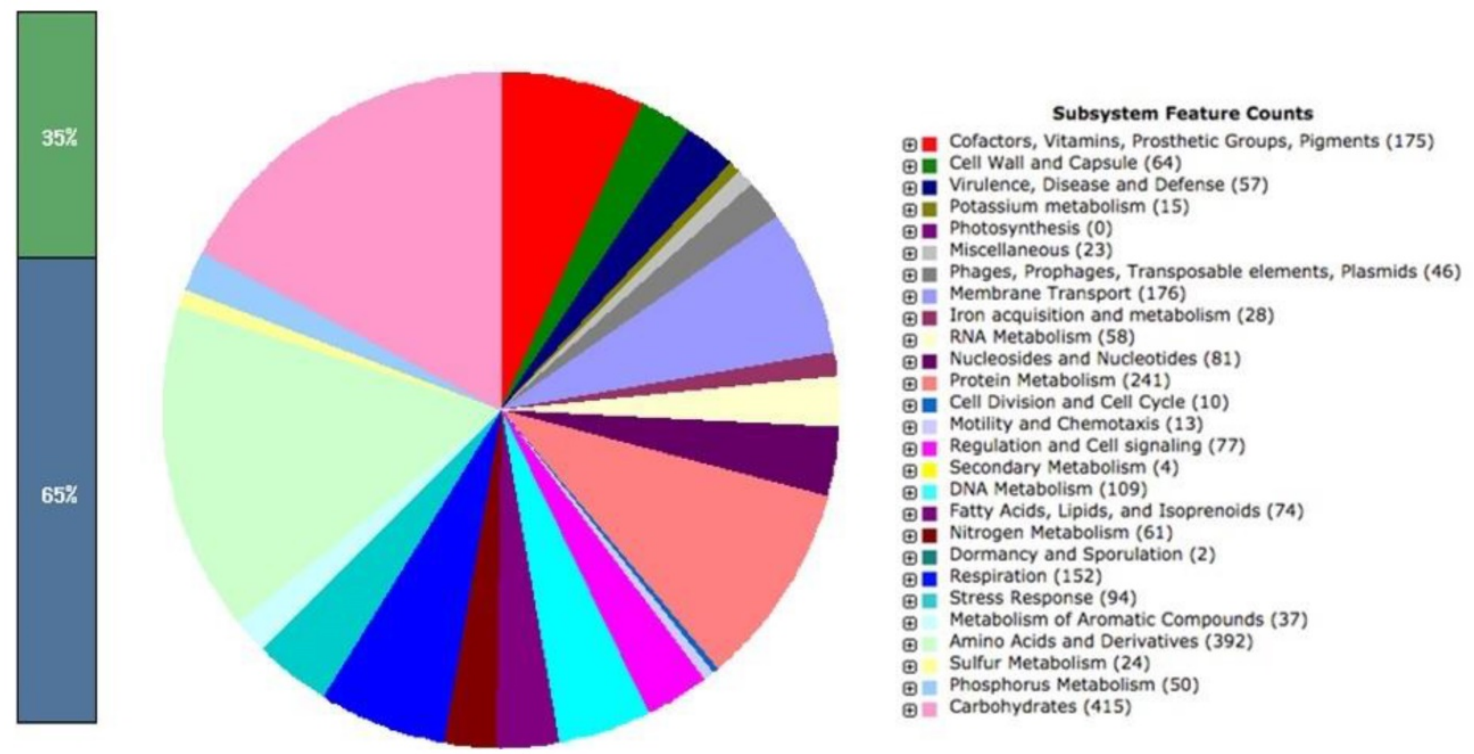

Figure 2. Salmonella Oslo (LAT9) subsystem features.

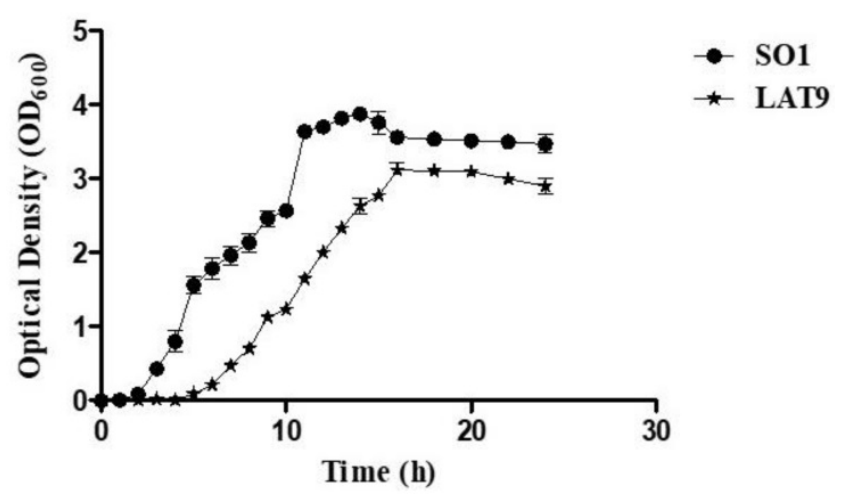

Figure 3. Growth kinetics of SOI and LAT9.

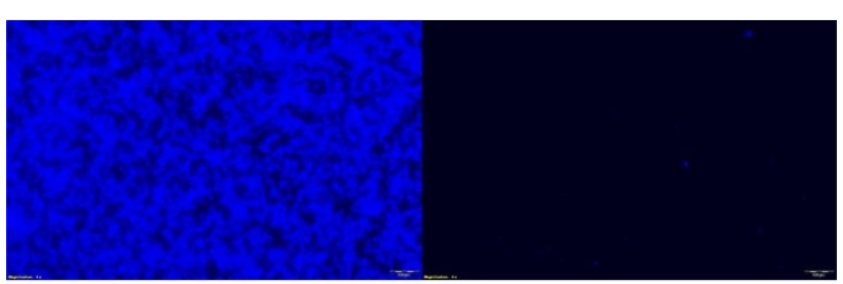

(A)

(B)

Figure 4. Calcoflour stained images of SOI (A) and LAT9 (B) strains under fluorescent microscope.

Further, the growth kinetic analysis revealed that the growth of LAT9 was significantly ( $p$-value of $>0.001$ ) slower than SO1 (Fig 3). The biofilm forming ability of LAT9 was also significantly reduced when compared with SO1 (Fig 4).

To the best of our knowledge, this is the first draft genome sequence of Salmonella Oslo isolated from seafood and its auxotrophic mutant LAT9. Determination of anti-cancer activity of this laboratory generated auxotrophic mutant using cell line and animal models would provide a suitable alternative to $S$. Typhimurium VNP20009 as candidate strains for bacteria-mediated anticancer therapy.

The whole genome shotgun projects have been submitted to GenBank and the assigned accession numbers are as follows: NZ_SJXK00000000 for SO1 and NZ_SMLR00000000 for LAT9. The version described in the paper represents the first version.

\section{Acknowledgement}

Financial support received from Nitte (Deemed to be University) and EMBO in collaboration with UCD (Dr. Seamus Fanning's Laboratory, Centre for food safety and zoonoses, Dublin) to the corresponding author is gratefully acknowledged.

\section{Funding}

The corresponding author has received financial support for this study from DST-SERB, Government of India in the form of an extramural grant (Grant no. ECR/2017/ 000559) and from Nitte (Deemed to be University) in the form of an intramural grant (NUFR1/2016/19-04).

\section{Competing Interests}

The authors have declared that no competing interest exists.

\section{References}

1. Deekshit VK, Kumar BK, Rai P, Karunasagar I, Karunasagar I. Differential expression of virulence genes and role of gyrA mutations in quinolone resistant and susceptible strains of $\mathrm{S}$ almonella $\mathrm{W}$ eltevreden and Newport isolated from seafood. J Appl Microbiol. 2015;119(4):970-80. doi: 10.1111/jam.12924.

2. Chen G, Tang B, Yang BY, Chen JX, Zhou JH, Li JH, Hua ZC. Tumor-targeting Salmonella typhimurium, a natural tool for activation of prodrug $6 \mathrm{MePdR}$ and their combination therapy in murine melanoma model. Appl Microbiol Biotechnol. 2013;97(10):4393-401. doi: $10.1007 /$ s00253-012-4321-8. 
3. Broadway KM, Suh S, Behkam B, Scharf BE. Optimizing the restored chemotactic behavior of anticancer agent Salmonella enterica serovar Typhimurium VNP20009. J Biotechnol. 2017; 251:76-83. doi: 10.1016/j.jbiotec.2017.04.006.

4. Coutermarsh-Ott SL, Broadway KM, Scharf BE, Allen IC. Effect of Salmonella enterica serovar Typhimurium VNP20009 and VNP20009 with restored chemotaxis on $4 \mathrm{~T} 1$ mouse mammary carcinoma progression. Oncotarget. 2017;8(20):33601. doi: 10.18632/oncotarget.16830.

5. Yano S, Zhang Y, Zhao M, Hiroshima Y, Miwa S, Uehara F, Kishimoto H, Tazawa H, Bouvet M, Fujiwara T, Hoffman RM. Tumor-targeting Salmonella typhimurium A1-R decoys quiescent cancer cells to cycle as visualized by FUCCI imaging and become sensitive to chemotherapy. Cell Cycle. 2014;13(24):3958-63. doi: 10.4161/15384101.2014.964115.

6. Igarashi K, Kawaguchi K, Kiyuna T, Miyake K, Miyake M, Li S, Han Q, Tan Y, Zhao M, Li Y, Nelson SD. Tumor-targeting Salmonella typhimurium A1-R combined with recombinant methioninase and cisplatinum eradicates an osteosarcoma cisplatinum-resistant lung metastasis in a patient-derived orthotopic xenograft (PDOX) mouse model: decoy, trap and kill chemotherapy moves toward the clinic. Cell Cycle. 2018;17(6):801-9. doi: 10.1080/15384101.2018.1431596.

7. Fu W, Lan H, Li S, Han X, Gao T, Ren D. Synergistic antitumor efficacy of suicide/ePNP gene and 6-methylpurine 2'-deoxyriboside via Salmonella against murine tumors. Cancer Gene Therapy. 2008;15(7):474. doi: 10.1038/cgt.2008.19.

8. Jazeela K, Chakraborty A, Karunasagar I, Vijaya Kumar D. NonTyphoidal Salmonella: A Potential Anti-Cancer Agent. J Appl Microbiol. 2019. https://doi.org/10.1111/jam.14297

9. Pangilinan CR, Lee CH. Salmonella-Based Targeted Cancer Therapy: Updates on A Promising and Innovative Tumor Immunotherapeutic Strategy. Biomedicines. 2019;7(2):36. doi: 10.3390/biomedicines7020036.

10. Zhou S, Gravekamp C, Bermudes D, Liu K. Tumour-targeting bacteria engineered to fight cancer. Nat Rev Cancer. 2018:1. doi: 10.1038/s41568-018-0070-z.

11. Andrews WH, Hammack T. Salmonella. In Bacteriological Analytical Manual US Food and Drug Administration. Available from: http://www.fda.gov/Food/ScienceResearch/LaboratoryMethods/Bact eriologicalAnalytical ManualBAM/default.htm. 2011.

12. Deekshit VK, Kumar BK, Rai P, Srikumar S, Karunasagar I, Karunasagar I. Detection of class 1 integrons in Salmonella Weltevreden and silent antibiotic resistance genes in some seafood-associated nontyphoidal isolates of Salmonella in south-west coast of India. J Appl Microbiol. 2012;112(6):1113-22. doi: 10.1111/j.1365-2672.2012.05290.x.

13. Datsenko KA, Wanner BL. One-step inactivation of chromosomal genes in Escherichia coli K-12 using PCR products. Proceedings of the National Academy of Sciences. 2000;97(12):6640-5. DOI: 10.1073/pnas.120163297

14. Stepanović S, Ćirković I, Ranin L, S vabić-Vlahović M. Biofilm formation by Salmonella spp. and Listeria monocytogenes on plastic surface. Lett Appl Microbiol 2004; 38(5):428-32. DOI: 10.1111/j.1472-765X.2004. 01513.x

15. Founoua LL, Founoua RC, Allamd M, Ismail A, Essack SY. Draft genome sequence of a methicillin-resistant Staphylococcus epidermidis isolate from swine. J Glob Antimicrob Resist 2018;15: 250-251. DOI: 10.1016/j.jgar.2018.10.016

16. Aziz RK, Bartels D, Best AA, DeJongh M, Disz T et al. The RAST Server: rapid annotations using subsystems technology. BMC genomics. 2008; 9(1):75. https://doi.org/10.1186/1471-2164-9-75. 